\title{
Neighborhood-based PA and its environmental correlates: a GIS- and GPS based cross-sectional study in the Netherlands
}

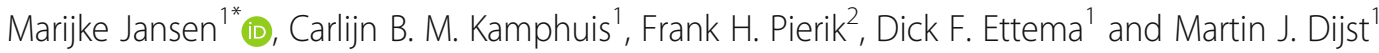

\begin{abstract}
Background: To improve our understanding of the neighborhood environment - physical activity (PA) relationship, it is of importance to assess associations between neighborhood environmental characteristics and neighborhoodbased PA.

Methods: Participants' ( $N=308 ; 45-65$ years) light PA (LPA) and moderate-vigorous PA (MVPA) within a 400, 800, and $1600 \mathrm{~m}$ buffer around adults' homes was measured using accelerometers and GPS-devices. Land use data in ArcGIS provided neighborhood characteristics for the same buffers. Multilevel linear regression models, adjusted for socio-demographic variables and attitude towards PA, were used to assess associations of objective neighborhood characteristics with neighborhood-based LPA and MVPA.

Results: LPA was positively associated with the proportions of roads (within a $400 \mathrm{~m}$ buffer), and negatively associated with the proportions of recreational areas (within an $800 \mathrm{~m}$ buffer), and the proportion of green space (within the $800 \mathrm{~m}$ and $1600 \mathrm{~m}$ buffers). Multiple characteristics of $400 \mathrm{~m}$ buffers were positively associated with MVPA, i.e. proportions of green space, blue space, residences, shops and foodservice industry, sports terrain, and public social-cultural facilities. Also, characteristics of larger buffers were positively associated with MVPA, i.e. the proportions of shops and foodservice industry, sports terrain, and blue space (within an $800 \mathrm{~m}$ buffer), and the proportion of public social-cultural facilities (within the $800 \mathrm{~m}$ and $1600 \mathrm{~m}$ buffers).
\end{abstract}

Conclusions: Objective neighborhood characteristics of smaller as well as larger sized buffers were associated with neighborhood-based LPA and MVPA. Green and blue spaces seem to be of particular importance for PA in the smallest buffer, i.e. in the direct surrounding of adults' homes.

Keywords: Neighborhood-based physical activity, GPS, Accelerometer, GIS, Objective neighborhood characteristics

\section{Background}

Regular physical activity (PA) positively affects health [1, 2]. To achieve health benefits from PA, the World Health Organization (WHO) recommends adults to engage in at least 150 min of moderate PA, or 75 min of vigorous PA per week, or an equivalent combination of both [3]. Worldwide $31.1 \%$ of the adult population is insufficiently

\footnotetext{
* Correspondence: f.m.jansen@uu.nl

${ }^{1}$ Department of Human Geography and Spatial Planning, Faculty of Geosciences, Utrecht University, Heidelberglaan 2, 3584 CS Utrecht, The Netherlands

Full list of author information is available at the end of the article
}

physically active [4], and increasing population levels of PA is of great importance for population health. However, it is not only moderate-vigorous PA (MVPA) that is of importance for health. Over the past years, researchers have increasingly emphasized the importance for activities of daily living (e.g. household activities, walking, and gardening) or light intensity activities (LPA) as well [5-7]. To adequately inform policy makers, intervention developers and urban planners in designing PA-friendly environments that facilitate both LPA and MVPA, it is important to better understand the relationship between environmental characteristics and PA. 
Daily life activities, including PA, take place in many different places (see e.g. [8]). Therefore, throughout the day, individuals are exposed to various environments that have different characteristics. One environment of interest is the residential environment. This environment is one of the daily life environments where individuals spend a great amount of their time (i.e. 60\% [9]). For example, the use of various services (e.g. banks, restaurants, and post offices) as well as daily (food) shopping, and other activities, such as walking the dog, or jogging may take place in the residential neighborhood.

The majority of studies investigating the relationship between neighborhood characteristics and PA have used selfreport methods to measure PA, and included outcomes such as total PA, leisure-time PA, walking, and cycling (see e.g. [10-12]). Although some neighborhood characteristics (e.g. walkability, land use mix) have been consistently associated with increased PA levels [11, 12], it is largely unknown whether neighborhood characteristics may also contribute to objectively measured LPA, and MVPA. Insight in these relationships may provide useful information to develop adequate interventions that aim to increase (a specific) intensity of PA through environmental changes.

In addition, many studies that investigated the role of neighborhood characteristics in PA behavior assessed the role of neighborhood characteristics on total PA levels (see e.g. [13-17]). These PA levels - whether they are walking, overall PA, or leisure time PA - often include both activities within and outside the residential neighborhood. However, this may lead to a conceptual mismatch of PA behavior and environmental exposure [18], which may underestimate the actual physical environment - PA association at the neighborhood level. Thus, there is a need for context-specific PA assessment, where neighborhood characteristics are matched with neighborhoodbased PA. One study that examined the association between neighborhood-based PA and neighborhood characteristics, found that higher levels of land use mix, intersection density, and residential population density, and residential housing unit density were positively related to MVPA within a $1 \mathrm{k}$ buffer around the home [19]. Although this provides useful insights, more specificity on the types of objectively measured land use can provide additional and more concrete evidence that contributes to the development of environmental interventions.

Therefore, the aim of this study was to investigate which objective neighborhood characteristics (i.e. types of land uses) are associated with neighborhood-based LPA and MVPA.

\section{Methods}

Aim, design and setting of the study

This cross-sectional study was part of the PHASE (Physical Activity in public Space Environments) project that aimed to investigate PA behavior in various environments and how environmental settings and their characteristics are related to PA behavior. Participants were randomly recruited from the municipal population register of the cities of Rotterdam and Maastricht, the Netherlands. Recruitment took place in two different cities to compose a study sample with varying environmental exposures (i.e. presence of green, distance to city center, type of buildings, and population density). A more detailed overview of differences in environmental characteristics between Rotterdam and Maastricht, and the four neighborhoods, were provided elsewhere [8]. Adults aged $45-65$ years $(N=14,889)$ received an invitation letter to ask them to participate in the study. Adults could register for participation via a website or by telephone. Those who registered $(N=516)$ were contacted by phone or e-mail to plan the distribution of an accelerometer and GPS-device. Trained staff members distributed devices and explained monitor wear to participants $(N=406)$ in community centers close to participants homes. Sheets with a summary of instructions were provided. Data collection took place from April to December 2014. All participants signed informed consent. One participant was excluded from analyses due to insufficient data on the home address. Only participants with sufficient valid accelerometer- and GPS-data were included in analyses. After applying valid data criteria (see below), 308 participants (with a total of 1804 measurement days) could be included in the analyses. The institutional review board of the faculty of Social and Behavioural Sciences of the Utrecht University approved for the study.

\section{Measures}

\section{Neighborhood-based PA}

Participants were asked to wear an accelerometer and GPS-device, which were attached to an elastic and adjustable belt, during waking hours for 7 consecutive days (except during water-based activities). The Actigraph GT3X+ accelerometer (Actigraph, Pensacola, Florida, FL, USA) was used to measure PA intensity. The epoch length was 5 s. Actilife v6.11.2 (Firmware 2.2.1, Actigraph, Pensacola, Florida, USA) software was used to download accelerometer data. As the study population was middle-aged, and therefore more likely to have longer bouts of sedentary behavior than younger adults non-wear time was defined as episodes of $\geq 90 \mathrm{~min}$ of consecutive zero counts, accepting up to 2 consecutive minutes of 1-100 cpm [20, 21]. Vector magnitude cutpoints were used to define light (150-3208 cpm), moderate $(3208-8564 \mathrm{cpm})$ and vigorous $(\geq 8565 \mathrm{cpm}) \mathrm{PA}$ $[22,23]$. Moderate-vigorous PA was calculated as the sum of moderate and vigorous PA.

BT-Q1000XT GPS-devices (QStarz International Co, Taipei, Taiwan) were used to measure participants' 
geographical locations every $5 \mathrm{~s}$. The GPS-device was attached to the same belt the accelerometer was attached to. The QStarz QTravel software (v1.45, Qstarz International Co., Ltd., Taipei, Taiwan) was used to download the data. For each GPS data-point it was determined whether it lay within a $25-400,25-800$, or $25-1600 \mathrm{~m}$ Euclidian buffer around participants' homes. We applied the $>25 \mathrm{~m}$ criterion for each neighborhood buffer to exclude the time spent at home. We first calculated the percentage of time spent within each buffer. In addition, GPS - and accelerometer data were date and time linked (using python software), and this combination of data was used to determine the proportion of time spent on light intensity activities (LPA) and moderate-vigorous intensity activities (MVPA) in each buffer. These percentages of time spent on LPA and MVPA in three different buffers (i.e. $400 \mathrm{~m}, 800 \mathrm{~m}$, and $1600 \mathrm{~m}$ ) were used as the outcome measures in the analyses.

\section{Valid days}

A valid day was determined using the $70 / 80$ rule [24]. Therefore, we first determined a measurement day, which is the time during which at least $70 \%$ of participants wore the accelerometer devices. For this study, the length of a measurement day was $611 \mathrm{~min}$. The 70/80 rule defines a day to be valid when at least $80 \%$ of a measurement day has non-missing counts, which was $488.8 \mathrm{~min}$ for this study. Data of participants with at least 4 valid days were included in analyses [25].

\section{Objective neighborhood characteristics}

The coordinates of the home addresses of participants were uploaded in ArcMap. A 400, 800, and $1600 \mathrm{~m} \mathrm{Eu-}$ clidean buffer (drawn around each home address) was used to define participants' neighborhoods. The proportions of different types of land use (available from Statistics Netherlands, 2012) were calculated for each of these buffers. Nine categories of land use were distinguished: residences, roads, public social and cultural facilities (e.g. educational institutes, churches), shops and food service industry (e.g. shopping centers, cinemas, hotels, restaurants), blue space (i.e. the sum of all proportions of visible surface waters e.g. rivers, lakes, recreational pools in forests, sea), green space (i.e. the sum of all proportions of green such as city parks, allotments, forests, moorland), sports terrain (e.g. football fields, tennis courts, swimming pool, sports hall), and recreational area (e.g. picnic places, zoo). In this paper, neighborhood characteristics thus refer to the proportions of different types of land use (characteristics) in a 400, 800, and $1600 \mathrm{~m}$ buffer around participants' homes (neighborhood). Although these types of land use covered most of the land use types that were found within buffers, there were some types of land use that were not included in the analyses because these proportions were very low. This included for example cemeteries, or dumps.

\section{Individual factors}

A questionnaire was used to collect data on background variables (e.g. age, gender, ethnicity, and education), the home address, and attitude towards PA. Self-reported highest levels of completed education were classified into three levels: 1) lower education (i.e. no education, primary education, lower professional or intermediate general education); 2) middle education (i.e. intermediate and higher general education); and 3) higher education (i.e. higher professional education and university). Attitude was measured by asking participants to indicate on a 5-point Likert scale to what extent they agreed with four statements: PA is good for me, PA is pleasant, PA is important and PA gives variation. These variables were aggregated into the variable 'attitude' by summing the scores of the separate items (Cronbach's alpha: 0.870, this value did not increase if items were deleted).

\section{Statistical analyses}

All analyses were performed using SPSS 23.0 for windows (IBM SPSS Inc., Armonk, NY). Descriptive statistics were used to present data on population- and neighborhood characteristics, and the amounts of time spent within neighborhood buffers. To assess the role of neighborhood characteristics (independent variables) in neighborhood-based LPA and MVPA (outcome variables), bootstrapped multilevel linear regression analyses were performed. Regressions were bootstrapped because the outcome variables were not normally distributed and neither log transformations nor taking the square root led to normal distributions. Multilevel analyses were used to consider the multilevel structure of the data: days were organized within respondents (and days of one respondent are more similar to each other than to those of other respondents). Analyses were adjusted for age, gender, BMI (Body Mass Index), education, ethnicity, having a car, having children, dog ownership, city of residence (Rotterdam or Maastricht), and attitude towards PA.

\section{Results}

\section{Descriptive statistics}

Of the total study population $(N=308)$, a little more than half was female (Table 1). Adults were on average 56.4 (SD 6.2) years, over $60 \%$ were employed, most adults had a middle or higher education, and more than $80 \%$ of the population was native Dutch. About $1 / 3$ of the study population had at least one child, and approximately $1 / 5$ had a dog. The most common type of land use of the buffers surrounding participants' homes was residences (Table 2 ). 
Table 1 Descriptive statistics of the study population $(N=308)$

\begin{tabular}{|c|c|}
\hline \multicolumn{2}{|l|}{ Individual factors } \\
\hline Age in years Mean ( $\pm S D$ ) & $56.4( \pm 6.2)$ \\
\hline Female \% & 54.9 \\
\hline \multicolumn{2}{|l|}{ BMI \% } \\
\hline Healthy weight & 52.9 \\
\hline Overweight & 37.0 \\
\hline Obesity & 10.1 \\
\hline \multicolumn{2}{|l|}{ Education \% } \\
\hline Low & 4.2 \\
\hline Middle & 53.2 \\
\hline High & 40.9 \\
\hline Missing & 1.6 \\
\hline \multicolumn{2}{|l|}{ Ethnicity \% } \\
\hline Autochthonous & 84.4 \\
\hline Western immigrants & 6.8 \\
\hline Non-western immigrants & 7.5 \\
\hline Missing & 1.3 \\
\hline \multicolumn{2}{|l|}{ Having a dog $\%$} \\
\hline Yes & 18.8 \\
\hline Missing & 0.6 \\
\hline \multicolumn{2}{|l|}{ Having children \% } \\
\hline Yes & 33.1 \\
\hline \multicolumn{2}{|l|}{ Having a car $\%$} \\
\hline Yes & 82.8 \\
\hline Missing & 0.6 \\
\hline \multicolumn{2}{|l|}{ City \% } \\
\hline Rotterdam & 38.0 \\
\hline Maastricht & 62.0 \\
\hline \multicolumn{2}{|l|}{ Attitude (score) } \\
\hline Mean ( \pm SD) & $18.0(2.4)$ \\
\hline Missing (\%) & 0.3 \\
\hline
\end{tabular}

Note: $S D$ standard deviation, $B M I$ Body Mass Index, IQR interquartile range ${ }^{\text {a}}$ This includes the time spent at home. ${ }^{\text {b }}$ Excluding the time spent at home

Participants spent on average $29.1 \%$ of a measurementday in LPA, and $5.7 \%$ in MVPA (Table 3). Participants spent more time outside the 400, 800, and $1600 \mathrm{~m}$ buffers than within the buffers. The average percentage of LPA and MVPA within the buffers is approximately similar to LPA and MVPA outside the buffers.

\section{The role of neighborhood characteristics in LPA and MVPA within the neighborhood}

Various objective neighborhood characteristics were significantly associated with LPA and MVPA within the neighborhood (Table 4). Neighborhood characteristics that were associated with LPA were different from the neighborhood characteristics that were associated with MVPA. Also, different significant associations were found for buffers of different sizes.

The proportion of roads was positively associated with LPA within a $400 \mathrm{~m}$ buffer. The proportions of recreational area and green space were negatively associated with LPA within an $800 \mathrm{~m}$ buffer, and the proportion of green space was negatively associated with LPA within a $1600 \mathrm{~m}$ buffer.

With regard to MVPA, positive associations were found between the proportions of residences, shops and foodservice industry, sports terrain, public socialcultural facilities, green space, and blue space and MVPA within a $400 \mathrm{~m}$ buffer, whereas the proportion of recreational area was negatively associated with MVPA within a $400 \mathrm{~m}$ buffer. The proportions of shops and foodservice industry, sports terrain, public socialcultural facilities, and blue space were positively associated with MVPA within an $800 \mathrm{~m}$ buffer. Further, the proportion of public social-cultural facilities was positively associated with MVPA within a $1600 \mathrm{~m}$ buffer.

\section{Discussion}

This study found significant associations between objective neighborhood characteristics and neighborhood-based LPA and MVPA, also when adjusted for an extensive set of individual factors, including attitude towards PA. This makes it less likely that the correlation between neighborhood characteristics and neighborhood-based PA is purely a matter of selection (i.e. that those with a more favorable attitude towards $\mathrm{PA}$, and practicing more $\mathrm{PA}$, chose to reside in neighborhoods that facilitate $\mathrm{PA})$, which may indicate that a causal mechanism underlies the correlations found in this study.

The current study showed that more neighborhood characteristics were associated with MVPA than with LPA. A possible explanation for this finding may be that LPA is often part of everyday activities (e.g. household activities, walking), which are integrated in adults' daily activity patterns. That is, these activities may be more likely to occur in any case, whereas MVPA may require more planning, skills, motivation, and specific facilities or environmental features.

Additionally, most effects are found for the smallest buffer around the home (i.e. $400 \mathrm{~m}$ buffer), whereas less (i.e. for MVPA) or even negative (i.e. for LPA) associations were found for the larger buffers. This suggests that objective neighborhood characteristics may be of particular importance for PA in the area directly surrounding adults' homes. Further, these findings emphasize that size of a buffer matters when assessing the physical environment PA relationship. Hence, future studies should consider the use of multiple buffers when assessing the relationship 
Table 2 Proportions of participants' ( $\mathrm{N}=308$ ) with certain neighborhood characteristics (\% land use) in different buffers surrounding their homes

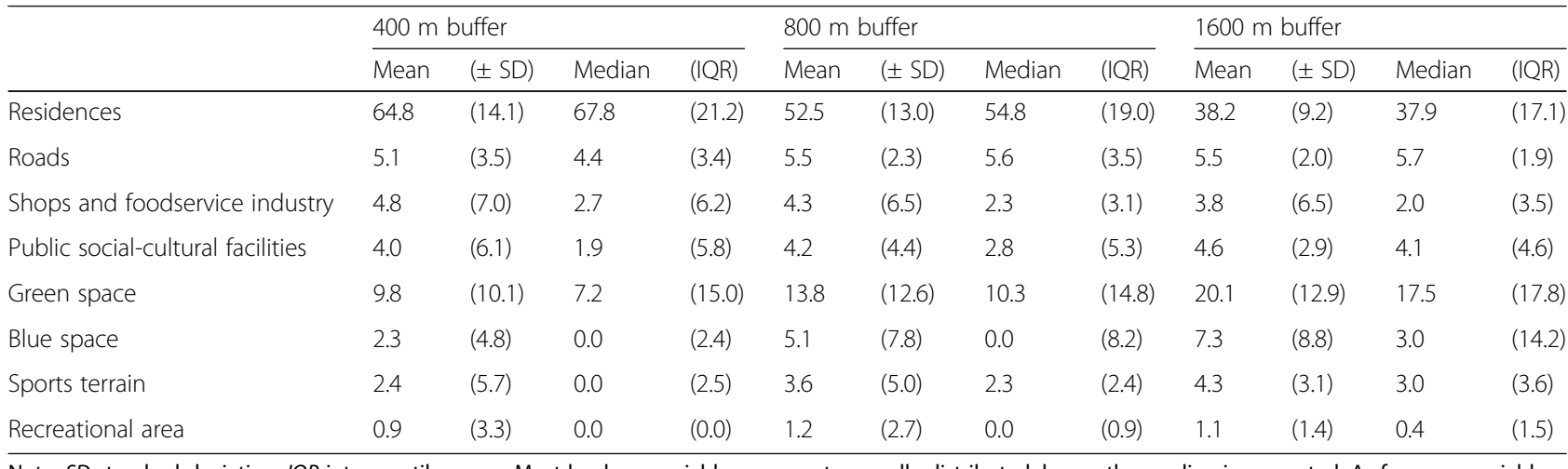

Note: $S D$ standard deviation, IQR interquartile range. Most land use variables were not normally distributed, hence the median is presented. As for some variables the median was 0 , the mean was also presented for interpretation

between neighborhood characteristics and PA, or choose a buffer that fits specific policy or urban design aims.

The importance of environmental characteristics close to individuals' homes seems to apply in particular to natural environments (i.e. green and blue spaces), as the positive associations found for MVPA within a $400 \mathrm{~m}$ buffer disappear when buffer size increases. Moreover, negative associations were found between green space and LPA within the 800 and $1600 \mathrm{~m}$ buffers. A possible explanation for this may be that adults are more familiar with green and blue spaces within a shorter distance from their home than with green and blue spaces further away. Also, the use of green and blue spaces on short distance to the home is easier to integrate with obligatory activities during the day. With the current findings, this study expands existing literature that found that amount and size of, and distance to urban green space, play an important role in stimulating PA behavior [26, 27], by demonstrating that this effect emerges via PA close to the residence. Also, the positive effects of blue spaces on physical activities such as walking (the dog), jogging or cycling at the water's edge [28], apparently occur mostly on short distance to the home.

Proportions of roads was the only type of land use that was positively associated with LPA, and only within a
$400 \mathrm{~m}$ buffer. An explanation for this may be that an increased proportion of roads is related to increased walkability and connectivity, and a reduced distance to side-walks or trails; factors that have been positively related to walking (i.e. an important source of LPA [7]) and PA in previous studies (see e.g. [10-12]). Also, findings that higher proportions of sports terrain, public social-cultural facilities, and shops and foodservice industry were positively associated with MVPA are in line with existing literature that showed, although not at the level of neighborhood-based MVPA, that sports facilities [29] and presence and accessibility of shops and other facilities (see e.g. [10, 30]) were associated with (MV)PA.

Perhaps less expected -at first sight- was the finding that a higher proportion of recreational areas within one's residential neighborhood was associated with lower levels of LPA within an $800 \mathrm{~m}$ buffer and MVPA within a $400 \mathrm{~m}$ buffer. However, in this study, the land use category of 'recreational area' did not include parks or sports facilities, which belonged to other types of land uses (i.e. green space and sports facilities, respectively). In the current study, 'recreational area' refers to places such as zoos, amusement parks, open-air museums, playgrounds, and picnic places. Such places likely facilitate mostly sedentary behavior (e.g. social activities) and

Table 3 Daily percentages of total time, light PA, and moderate-vigorous PA spent in different buffers

\begin{tabular}{|c|c|c|c|c|c|c|}
\hline & \multicolumn{2}{|c|}{ Total time \% } & \multicolumn{2}{|l|}{ LPA \% } & \multicolumn{2}{|c|}{ MVPA \% } \\
\hline & Median & $I Q R$ & Median & $I Q R$ & Median & $I Q R$ \\
\hline Total & & & 29.1 & $(22.6 ; 35.9)$ & 5.7 & $(3.4 ; 8.9)$ \\
\hline At home $(0-25 \mathrm{~m})$ & 40.5 & $(19.1 ; 62.8)$ & 27.6 & $(19.8 ; 36.0)$ & 3.9 & $(2.4 ; 6.6)$ \\
\hline Within a 25-400 m buffer & 11.6 & $(4.9 ; 24.0)$ & 27.9 & $(19.3 ; 38.2)$ & 4.5 & $(2.1 ; 9.6)$ \\
\hline Within a $25-800 \mathrm{~m}$ buffer & 13.8 & $(6.4 ; 28.6)$ & 28.6 & $(20.1 ; 38.9)$ & 4.6 & $(2.2 ; 10.0)$ \\
\hline Within a 25-1600 m buffer & 19.8 & $(9.4 ; 36.3)$ & 30.0 & $(21.4 ; 38.6)$ & 5.1 & $(2.5 ; 10.3)$ \\
\hline$>1600 \mathrm{~m}$ from the home & 24.2 & $(5.1 ; 55.7)$ & 28.6 & $(21.9 ; 35.9)$ & 5.0 & $(3.0 ; 8.2)$ \\
\hline
\end{tabular}

Note: Percentages represent daily averages of measurement days. Percentages in columns (e.g. at home, within 25-400 m, and $>400 \mathrm{~m}$ ) do not necessarily add up to $100 \%$ because medians are presented. Medians were presented due to non-normality. LPA Light PA, MVPA Moderate-Vigorous PA 
Table 4 Associations between neighborhood characteristics and percentage of LPA and MVPA within the neighborhood

\begin{tabular}{|c|c|c|c|c|c|c|}
\hline & $B$ & $95 \% \mathrm{Cl}$ & $p$ & $B$ & $95 \% \mathrm{Cl}$ & $p$ \\
\hline & \multicolumn{3}{|c|}{$\%$ LPA within a 25-400 m buffer } & \multicolumn{3}{|c|}{$\%$ MVPA within a 25-400 m buffer } \\
\hline \multicolumn{7}{|c|}{ Neighborhood characteristics within a 25-400 m buffer } \\
\hline Intercept & 26.18 & & & 9.54 & & \\
\hline Residences & 0.01 & $(-0.07 ; 0.09)$ & 0.847 & 0.05 & $(0.01 ; 0.10)$ & 0.010 \\
\hline Roads & 0.20 & $(-0.01 ; 0.42)$ & 0.032 & 0.02 & $(-0.13 ; 0.16)$ & 0.802 \\
\hline Shops and foodservice industry & 0.04 & $(-0.09 ; 0.16)$ & 0.481 & 0.15 & $(0.07 ; 0.23)$ & 0.001 \\
\hline Sports terrain & 0.04 & $(-0.09 ; 0.17)$ & 0.468 & 0.08 & $(0.00 ; 0.17)$ & 0.023 \\
\hline Public social-cultural facilities & 0.03 & $(-0.10 ; 0.18)$ & 0.590 & 0.06 & $(-0.01 ; 0.12)$ & 0.042 \\
\hline Recreational area & -0.17 & $(-0.39 ; 0.06)$ & 0.080 & -0.12 & $(-0.23 ;-0.03)$ & 0.005 \\
\hline Green space & -0.08 & $(-0.19 ; 0.02)$ & 0.083 & 0.11 & $(0.02 ; 0.19)$ & 0.001 \\
\hline \multirow[t]{2}{*}{ Blue space } & 0.10 & $(-0.08 ; 0.27)$ & 0.187 & 0.19 & $(0.10 ; 0.28)$ & 0.001 \\
\hline & \multicolumn{3}{|c|}{$\%$ LPA within a $25-800 \mathrm{~m}$ buffer } & \multicolumn{3}{|c|}{$\%$ MVPA within a $25-800 \mathrm{~m}$ buffer } \\
\hline \multicolumn{7}{|c|}{ Neighborhood characteristics within a 25-800 m buffer } \\
\hline Intercept & 25.96 & & & 7.88 & & \\
\hline Residences & 0.05 & $(-0.03 ; 0.12)$ & 0.172 & 0.03 & $(-0.02 ; 0.09)$ & 0.166 \\
\hline Roads & -0.05 & $(-0.36 ; 0.22)$ & 0.682 & -0.00 & $(-0.19 ; 0.19)$ & 0.983 \\
\hline Shops and foodservice industry & 0.11 & $(-0.05 ; 0.25)$ & 0.118 & 0.09 & $(-0.00 ; 0.18)$ & 0.034 \\
\hline Sports terrain & -0.02 & $(-0.17 ; 0.13)$ & 0.707 & 0.15 & $(0.04 ; 0.27)$ & 0.004 \\
\hline Public social-cultural facilities & -0.01 & $(-0.19 ; 0.18)$ & 0.911 & 0.12 & $(0.04 ; 0.21)$ & 0.003 \\
\hline Recreational area & -0.30 & $(-0.63 ; 0.04)$ & 0.047 & -0.16 & $(-0.35 ; 0.07)$ & 0.095 \\
\hline Green space & -0.09 & $(-0.18 ; 0.00)$ & 0.032 & 0.04 & $(-0.02 ; 0.11)$ & 0.136 \\
\hline \multirow[t]{2}{*}{ Blue space } & 0.12 & $(-0.02 ; 0.27)$ & 0.057 & 0.08 & $(-0.01 ; 0.16)$ & 0.046 \\
\hline & \multicolumn{3}{|c|}{$\%$ LPA within a $25-1600 \mathrm{~m}$ buffer } & \multicolumn{3}{|c|}{$\%$ MVPA within a 25-1600 m buffer } \\
\hline \multicolumn{7}{|c|}{ Neighborhood characteristics within a 25-1600 m buffer } \\
\hline Intercept & 29.35 & & & 11.53 & & \\
\hline Residences & 0.03 & $(-0.08 ; 0.16)$ & 0.567 & -0.00 & $(-0.09 ; 0.08)$ & 0.905 \\
\hline Roads & -0.31 & $(-1.29 ; 0.69)$ & 0.465 & -0.40 & $(-1.10 ; 0.28)$ & 0.166 \\
\hline Shops and foodservice industry & -0.14 & $(-0.43 ; 0.15)$ & 0.289 & -0.02 & $(-0.19 ; 0.16)$ & 0.824 \\
\hline Sports terrain & -0.10 & $(-0.52 ; 0.34)$ & 0.591 & 0.04 & $(-0.27 ; 0.35)$ & 0.787 \\
\hline Public social-cultural facilities & 0.16 & $(-0.32 ; 0.68)$ & 0.487 & 0.38 & $(0.03 ; 0.72)$ & 0.015 \\
\hline Recreational area & 0.23 & $(-0.61 ; 0.99)$ & 0.508 & 0.07 & $(-0.42 ; 0.65)$ & 0.787 \\
\hline Green space & -0.08 & $(-0.16 ; 0.01)$ & 0.045 & 0.02 & $(-0.04 ; 0.08)$ & 0.394 \\
\hline Blue space & -0.06 & $(-0.27 ; 0.15)$ & 0.497 & -0.06 & $(-0.19 ; 0.07)$ & 0.315 \\
\hline
\end{tabular}

Note. All models were adjusted for: age, gender, BMI, education, ethnicity, having a car, having children, dog ownership, city, and attitude towards PA. LPA Light PA, MVPA Moderate-Vigorous PA, CI Confidence Interval

Bolded text highlights significant associations

not so much LPA or MVPA, especially among this middle-aged study sample.

\section{Strengths and limitations}

The use of accelerometers and GPS-devices provided accurate and detailed information on neighborhood-based LPA and MVPA levels of adults. In addition, the use of objective land use information added to existing literature that mostly reported perceived environmental factors and/or observed environmental factors [12].
Furthermore, we included an extensive set of individual factors in analyses to correct for possible confounding effects (e.g. those with certain individual characteristics may select PA promoting neighborhoods).

The use of accelerometers and GPS-devices also has limitations. For example, upper-body movements are less well recorded by the accelerometer and water-based activities (i.e. swimming) could not be measured [31]. GPS-devices may suffer from canyoning (i.e. high buildings, or trees interfere with satellite communication), 
but the QStarz GPS-device that was used in this study has shown to have a high accuracy even in urban canyons [32]. Moreover, the use of GPS-devices and accelerometers often comes with relatively smaller study populations as compared to the use of questionnaires. However, although the response rate of this study was relatively low, our final study sample was comparable to other studies [12].

Finally, it is likely that a selective sample, i.e. adults who are interested in PA and like being active, responded to the invitation to participate in this study. Hence, more active adults may be included in this study, which may have led to an overestimation of PA levels. In addition, when comparing the figures of our sample with those of the total Dutch population, we see that overweight and obese adults, lower educated adults, and (non-) western immigrants were underrepresented in the study sample. Since the literature shows inconsistent findings on the correlations of these characteristics with PA (i.e. positive, negative, as well as null associations see e.g. [33, 34]), it remains unclear whether these under representations affected our results.

The inclusion of two different cities in the Netherlands contributed to differences in exposure to neighborhood environmental characteristics. Therefore, the findings of this study are more likely to be representative for the Netherlands. However, the findings of this study may not be applicable to other counties as urban design and environmental characteristics of residential areas (e.g. walking and cycling facilities, size of green spaces) may be very different between countries. In addition, the results of this study were found for a specific age group and future research is needed to assess what objectively measured neighborhood characteristics are related to neighborhood-based LPA and MVPA of other age groups (e.g. youth or older adults).

Due to the cross-sectional design of this study, only associations could be assessed and not causal relations. Future research should apply longitudinal, and preferably pre-posttest designs to investigate causal relationship between such neighborhood characteristics and PA.

\section{Conclusions}

This study responded to the need for more context-specific PA assessment by providing new insights in the role of objective neighborhood characteristics in neighborhood-based LPA and MVPA. Two main conclusions can be drawn from this study: 1) objective neighborhood characteristics play an important role in neighborhood-based PA, also when adjusted for socio-demographic factors and attitude towards PA. Hence, associations between the residential environment and PA found in previous studies can at least partly be explained by the effects on PA in the neighborhood, and 2) these neighborhood characteristics seem to be of particular importance for PA in the areas close to adults' homes (i.e. the smallest buffer around the home). Hence, size of the buffer matters when assessing the relationship between the residential environment and PA. Longitudinal pre-posttest study designs are necessary to assess the causality of the associations between objective neighborhood characteristics and objectively measured PA.

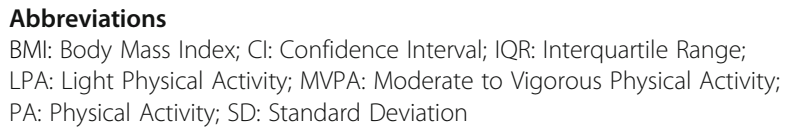

\section{Acknowledgements}

The authors thank the research assistants who helped collecting the data.

\section{Funding}

The Netherlands Organization for Scientific Research funded this project (project number: 328-98-005)

\section{Availability of data and materials}

The datasets generated during and/or analyzed during the current study are not publicly available as individual privacy could be compromised but are available from the corresponding author on reasonable request.

\section{Authors' contributions}

MJ drafted the manuscript and performed statistical analyses. DE, CK and FP participated in the design of the study. DE provided support and expertise in performing statistical analyses. DE, FP, CK and MD helped to draft the manuscript and critically reviewed the manuscript. All authors read the final manuscript and approved for publication.

\section{Ethics approval and consent to participate}

The study was conducted with approval of the institutional review board of the faculty of Social and Behavioural Sciences of the Utrecht University. All participants signed informed consent.

Consent for publication

Not applicable

\section{Competing interests}

All authors declare that they have no competing interests.

\section{Publisher's Note}

Springer Nature remains neutral with regard to jurisdictional claims in published maps and institutional affiliations.

\section{Author details}

'Department of Human Geography and Spatial Planning, Faculty of Geosciences, Utrecht University, Heidelberglaan 2, 3584 CS Utrecht, The Netherlands. ${ }^{2}$ Department of Sustainable Urban Mobility and Safety, TNO, P.O. Box 80015, 3508 TA Utrecht, The Netherlands.

Received: 23 January 2017 Accepted: 16 January 2018

Published online: 09 February 2018

\section{References}

1. Kruk J. Physical activity and health. Asian Pac J Cancer Prev. 2009;10:721-8.

2. Bize R, Johnson JA, Plotnikoff RC. Physical activity level and health-related quality of life in the general adult population: A systematic review. Prev Med. 2007; doi:https://doi.org/10.1016/j.ypmed.2007.07.017.

3. World Health Organization. Global Recommendations on Physical Activity for Health. Geneva: World Health Organization, WHO Press. 2010;59p. http:// apps.who.int/iris/bitstream/10665/44399/1/9789241599979_eng.pdf. Accessed 19 Jul 2016.

4. Hallal PC, Andersen LB, Bull FC, Guthold R, Haskell W, Ekelund U, et al. Global physical activity levels: surveillance progress, pitfalls, and prospects. Lancet. 2012;380:247-57. 
5. Howard B, EAH W, Sethi P, Carson V, Ridgers ND, Salmon J, et al. Associations of low- and high-intensity light activity with Cardiometabolic biomarkers. Med Sci Sports Exerc. 2015;47(10):2093-101.

6. Buman MP, Hekler EB, Haskell WL, Pruitt L, Conway $T L$, Cain $\mathrm{KL}$, et al. Objective light-intensity physical activity associations with rated health in older adults. Am J Epidemiol. 2010;172(10):1155-65.

7. Füzéki $E$, Banzer W. Activities of daily living and health. Public Health Forum. 2013;21(2):4.e1-4.

8. Jansen FM, Ettema DF, Pierik FH, Dijst MJ. Sports facilities shopping centers or homes: what locations are important for adults' physical activity? A crosssectional study. Int J Environ Res Public Health. 2016;13(3):287.

9. Mclnerney M, Csizmadi I, Friedenreich CM, Uribe FA, Nettel-Aguirre A, McLaren L, et al. Associations between the neighbourhood food environment, neighbourhood socioeconomic status, and diet quality: an observational study. BMC Public Health. 2016;16(1)

10. Badland H, Schofield G. Transport, urban design, and physical activity: an evidence-based update. Transp Res Part D: Transp Environ. 2005;10:177-96.

11. McCormack GR, Shiell A. In search of causality: A systematic review of the relationship between the built environment and physical activity among adults. International Journal of Behavioral Nutrition and Physical Activity. 2011; doi:https://doi.org/10.1186/1479-5868-8-125.

12. Van Holle V, Deforche B, Van Cauwenberg J, Goubert L, Maes L, Van de Weghe N, De Bourdeaudhuij. Relationship between the physical environment and different domains of physical activity in European adults: a systematic review. BMC Public Health. 2012; doi:https://doi.org/10.1186/ 1471-2458-12-807.

13. Carlson JA, Sallis JF, Conway TL, Saelens BE, Frank LD, Kerr J, ..., King AC. Interactions between psychosocial and built environment factors in explaining older adults' physical activity. Prev Med 2012 1/1;54(1):68-73.

14. Cerin E, Cain KL, Conway TL, Van Dyck D, Hinckson E, Schipperijn J, Sallis JF. Neighborhood environments and objectively measured physical activity in 11 countries. Med Sci Sports Exerc. 2014:46:2253-64.

15. Forsyth A, Michael Oakes J, Lee B, Schmitz KH. The built environment, walking, and physical activity: is the environment more important to some people than others? Transportation research part D. Transport and Environment. 2009;14(1):42-9.

16. Chudyk AM, McKay HA, Winters M, Sims-Gould J, Ashe MC. Neighborhood walkability, physical activity, and walking for transportation: a cross-sectiona study of older adults living on low income. BMC Geriatr. 2017;17(1)

17. Troped PJ, Tamura K, MH MD, Starnes HA, James P, Ben-Joseph E, et al. Direct and indirect associations between the built environment and leisure and utilitarian walking in older women. Ann Behav Med. 2017;51(2):282-91.

18. Matthews SA, Yang T. Spatial polygamy and contextual exposures (SPACEs): promoting activity space approaches in research on place and health. Am Behav Sci. 2013;57(8):1057-81.

19. Troped PJ, Wilson JS, Matthews CE, Cromley EK, Melly SJ. The built environment and location-based physical activity. Am J Prev Med. 2010; 38(4):429-38.

20. Herrmann SD, Barreira TV, Kang M, Ainsworth BE. Impact of accelerometer wear time on physical activity data: a NHANES semisimulation data approach. Br J Sports Med. 2012:48:278-82.

21. Choi L, Ward SC, Schnelle JF, Buchowski MS. Assessment of wear/nonwear time classification algorithms for triaxial accelerometer. Med Sci Sports Exerc. 2012;44:2009-16

22. Santos-Lozano A, Santin-Medeiros F, Cardon G, Torres-Luque G, Bailón R Bergmeir C, Ruiz JR, Lucia A, Garatachea N. Actigraph GT3X: validation and determination of physical activity intensity cut points. Int J Sports Med. 2013;34:975-82

23. Carr LJ, Mahar MT. Accuracy of intensity and inclinometer output of three activity monitors for identification of sedentary behavior and light-intensity activity. J Obes. 2012;2012:460271. doi:https://doi.org/10.1155/2012/460271. Epub 2011 Nov 13.

24. Catellier DJ, Hannan PJ, Murray DM, Addy CL, Conway TL, Yang S, Rice JC. Imputation of missing data when measuring physical activity by accelerometry. Med Sci Sports Exerc. 2005;37:S555-62.

25. Bento T, Cortinhas A, Leitão JC, Mota MP. Use of accelerometry to measure physical activity in adults and the elderly. Rev Saude Publica. 2012:46:561-70.

26. Schipperijn J, Bentsen P, Troelsen J, Toftager M, Stigsdotter UK. Associations between physical activity and characteristics of urban green space. Urban Forestry and Urban Greening. 2013;12(1):109-16.
27. Kaczynski AT, Henderson KA. Environmental correlates of physical activity: a review of evidence about parks and recreation. Leis Sci. 2007;29:315-54.

28. Völker S, Kisteman T. The impact of blue space on human health and wellbeing - Salutogenetic health effects of inland surface waters: a review. Int J Hyg Environ Health. 2001;214:449-60.

29. Halonen Jl, Stenholm S, Kivimäki M, Pentti J, Subramanian SV, Kawachi I, Vahtera J. Is change in availability of sports facilities associated with change in physical activity? A prospective cohort study. Prev Med. 2015;73:10-4.

30. Moran M, Van Cauwenberg J, Hercky-Linnewiel R, Cerin E, Deforche B, Plaut $P$. Understanding the relationships between the physical environment and physical activity in older adults: A systematic review of qualitative studies. Int J Behav Nutr Phys Act. 2014;11:79 doi:https://doi.org/10.1186/1479-586811-79.

31. Ridgers ND, Fairclough S. Assessing free-living physical activity using accelerometry: practical issues for researchers and practitioners. European Journal of Sport Science. 2011;11:205-13.

32. Schipperijn J, Kerr J, Duncan S, Madsen T, Klinker CD, Troelsen J. Dynamic accuracy of GPS receivers for use in health research: a novel method to assess GPS accuracy in real-world settings. Emerg Technol Promote Evaluate Phys Act. 2014;2:21 doi:https://doi.org/10.3389/fpubh.2014.00021.

33. Bauman AE, Reis R, RS SJF, Wells JC, RJF L, Martin BW. Correlates of physical activity: why are some people physically active and others not? Lancet. 2012;380:258-71.

34. Choi J, Lee M, Lee J, Kang D, Choi J. Correlates associated with participation in physical activity among adults: a systematic review of reviews and update. BMC Public Health. 2017:17(1):356 doi:https://doi.org/10.1186/ s12889-017-4255-2.

\section{Submit your next manuscript to BioMed Central and we will help you at every step:}

- We accept pre-submission inquiries

- Our selector tool helps you to find the most relevant journal

- We provide round the clock customer support

- Convenient online submission

- Thorough peer review

- Inclusion in PubMed and all major indexing services

- Maximum visibility for your research

Submit your manuscript at www.biomedcentral.com/submit 